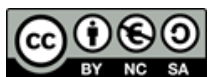

\title{
Sensibilización docente en procesos eficientes de inclusión educativa.
}

\section{Teacher awareness in efficient processes of educational inclusion}

\author{
Mercedes Jacqueline Aranda. ${ }^{1}$ \& Cecilia del Carmen Jiménez Sánchez. ${ }^{2}$
}

\begin{abstract}
.
Basis. Inclusion in education is a right of students with Special Educational NEEADds, when accessing formal educational institutions, the learning processes are complex due to multiple factors that are not consistent with the basic requirements to include them efficiently. One of the aspects of greatest incidence for inclusion is teacher awareness, which is an obstacle that is difficult to overcome when the institution lacks the mechanisms to improve the capacities of teachers. Objective. This scientific article was written with the objective of analyzing teacher awareness processes for the implementation of efficient educational inclusion. Methodology. It is a nonexperimental, descriptive research, based on the analysis of the results of an interview applied to 10 teachers linked to the inclusion processes of students with Special Educational NEEADds. Results. The first part of the work reviews the legal aspects related to educational inclusion and the mandatory nature of its application, emphasizing the duties and rights of the actors in the educational process, the second part of the article breaks down the opinion of the teachers interviewed considering the role of the institution in the inclusion processes; the treatment that should be given to the curriculum as an essential factor of sensitization for inclusion; It also deals with the ways in which the support to the students was carried out. The role of parents in the process was considered and the institutional resources to carry out educational inclusion were analyzed. All of this was discussed with the results of other investigations. Conclusions: The NEEADds for educational inclusion depend on a great diversity of factors that make this process

\footnotetext{
${ }^{1}$ Pontificia Universidad Católica del Ecuador, Maestría en Innovación en Educación, Tungurahua, mercedes.j.aranda@pucesa.edu.ec https://orcid.org/0000-0002-2924-5891

2 Pontificia Universidad Católica del Ecuador, Máster en Educación Especial, Bolívar, cajisan@hotmail.es https://orcid.org/0000-0002-9585-9728
} 
complex; Inclusion requires institutions to change infrastructure, resources and programmatic training for teacher awareness.

Key Words: Awareness, Special Educational NEEADds, Inclusion, Efficiency.

\section{Resumen.}

Fundamento. La inclusión en la educación es un derecho de los estudiantes con Necesidades Educativas Especiales asociados o no a una discapacidad, (NEEAD), al acceder a las instituciones educativas formales, los procesos de aprendizaje les resultan complejos por múltiples factores que no son consecuentes con los requerimientos básicos para incluirlos eficientemente. Uno de los aspectos de mayor incidencia para la inclusión es la sensibilización docente, que se constituye en un obstáculo difícilmente salvable cuando la institucionalidad carece de los mecanismos para mejorar las capacidades de los docentes. Objetivo. Este artículo científico fue escrito con el objetivo de analizar los procesos de sensibilización docente para la implementación de la inclusión educativa eficiente. Metodología. Es una investigación no experimental, descriptiva, se fundamenta en el análisis de los resultados de una entrevista aplicada a 10 docentes vinculados a los procesos de inclusión de estudiantes con Necesidades Educativas Especiales. Resultados. La primera parte del trabajo revisa los aspectos jurídicos vinculados a la inclusión educativa y la obligatoriedad de su aplicación, enfatizando en los deberes y derechos de los actores del proceso educativo, la segunda parte del artículo desglosa la opinión de los docentes entrevistados considerando el rol de la institución en los procesos de inclusión; el tratamiento que se le debe dar al currículo como factor esencial de sensibilización para la inclusión; se trata además, sobre las formas en las que se realizó el apoyo a los estudiantes, Se consideró el rol de los padres de familia en el proceso y se analizaron los recursos institucionales para llevar a cabo la inclusión educativa. Todo ello fue discutido con los resultados de otras investigaciones. Conclusiones. Las necesidades para la inclusión educativa dependen de una gran diversidad de factores que hacen complejo a este proceso; La inclusión requiere de las instituciones cambios de infraestructura, recursos y formación programática para la sensibilización docente.

Palabras Claves: Sensibilización, Necesidades Educativas Especiales, Inclusión, Eficiencia.

\section{Introducción.}

Desde la década de $1990^{3}$, se esperaba que los docentes ecuatorianos tuvieran una mayor responsabilidad de enseñar a los estudiantes con diversas discapacidades en aulas regulares y se esperó la consolidación de este proceso gracias a la Constitución de la República (2008) que habla de inclusión en mucho de sus aspectos, y la Normativa de estudiantes con necesidades educativas especiales (NEEAD) (MIES, 2013).

${ }^{3}$ Declaración de Salamanca en la Conferencia Mundial sobre Necesidades Educacionales Especiales, por la UNESCO en 1994 
Si bien la experiencia es un componente importante en el desarrollo de la competencia docente, otros problemas, por ejemplo, el nivel de conocimiento de los docentes sobre las diversas discapacidades y las actitudes hacia los estudiantes con NEEAD, también pueden influir en los resultados educativos de los estudiantes con este tipo de condición, como lo indican Acosta y Arráez (2017). Sin embargo, algunos enfoques tradicionales para dar información a los docentes de aula también pueden reducir o limitar sus sentimientos de competencia en relación con la enseñanza de estudiantes con diversas discapacidades (Martínez P \& Moreno P, 2018).

A la luz de la literatura reciente sobre de movimiento de inclusión algunos profesionales que tienen experiencia en la enseñanza y los roles administrativos en educación especial (Palacio S, 2019; Miranda V, Laz S, \& Campuzano P, 2020), han mantenido que las actitudes de los docentes hacia los estudiantes con necesidades educativas especiales son importantes factores en el éxito o fracaso de los intentos de incluir a estos niños en las aulas regulares. Por lo tanto, los programas de formación docente deben enseñar cuales deben ser las actitudes de los docentes hacia la inclusión de estudiantes con discapacidades en sus clases ampliando su base de información y sus habilidades de enseñanza. Además, los docentes podrían ser asesorados por docentes especialistas mientras trabajan con niños con necesidades educativas especiales (NEEAD) en sus aulas.

Fernández B (2018) propone que los docentes con actitudes positivas también deben tener cierta experiencia en la práctica docente para poder proporcionar las estrategias alternativas necesarias para los niños con discapacidades severas sugiere además que la preparación a los docentes es tan importante como el respaldo real para la inclusión que pueden proporcionar a los estudiantes en el aula. Si los docentes tienen resistencia a la inclusión, especialmente como se describe en la normativa legal (MIES, 2013), donde todavía puede confundirse fácilmente con el término "integración", puede constituir una barrera significativa para su implementación.

Según Palomino (2017), es deseable que los docentes tengan un curso de educación especial en todos los programas de formación docente previos al servicio para que desarrollen actitudes positivas hacia los estudiantes con NEEAD. En otras palabras, infundir información sobre NEEAD en los programas de formación docente puede ser un medio eficaz para cambiar las actitudes de manera positiva. Además, los docentes en servicio desean más cursos para enseñar a los niños con NEEAD, particularmente a los niños con discapacidades sensoriales, porque estos niños generalmente necesitan comunicación específica y apoyo técnico.

Las relaciones de colaboración entre los centros educativos y las instituciones de educación superior con especialidades en educación parecen ser necesarias para preparar mejor a los docentes para aceptar y apoyar a los estudiantes integrados en las aulas regulares con programas innovadores e informativos para enseñar a los niños con NEEAD (Olivares G \& García B, 2018). Los docentes que reciben capacitación regular en el servicio para enseñar a niños con NEEAD pueden tener actitudes más positivas hacia la 
inclusión que los docentes que no reciben información sobre educación especial (Herrera, et al, 2018).

Si los docentes reciben suficiente información y aplican lo que han aprendido en el aula real, pueden aprender a trabajar en colaboración con otros docentes y padres con beneficios para la inclusión. Además del desarrollo actitudinal, los docentes pueden ser más flexibles e ingeniosos y tener más estrategias que podrían usar para ayudar al estudiante, dentro y fuera del aula.

En la actualidad cada vez más docentes regulares se hacen responsables de la educación de los estudiantes con NEEAD en sus aulas, este aumento en la colocación de estudiantes con NEEAD en las escuelas convencionales es un resultado directo de la promulgación de la Normativa de estudiantes con necesidades educativas especiales (MIES, 2013). Aunque la práctica de la inclusión de estudiantes con NEEAD ha aumentado, se sabe poco sobre la situación que viven los docentes de aula regulares cuya responsabilidad se hace mayor al tener que educar a estos niños. La mayoría de ellos tienen inquietudes sobre los procesos de enseñanza que deben implementar y se perciben muchas limitaciones en sus capacidades y competencias docentes (Machado S \& Vintimilla C , 2019).

De acuerdo con lo establecido en la normatividad, los estudiantes con NEEAD deben incluirse tanto como sea posible en el aula habitual sin embargo, los materiales especiales, los servicios de apoyo y el equipo deben ponerse a disposición de los docentes para respaldar su inclusión (Lalama F, 2018), sumado a ello debería existir un grupo de docentes de apoyo, terapeutas y profesionales en procesos didácticos para las diferentes áreas de la atención a NEEAD como parte integral de los programas educativos en generales.

Los investigadores Sevilla, Martín y Jenaro (2018), no solo informaron que muchos educadores tienen actitudes negativas hacia la inclusión de estudiantes con NEEAD, sino que también enfatizaron la importancia de las actitudes positivas para el éxito de la inclusión. Las actitudes negativas de los docentes pueden crear expectativas de bajo rendimiento, mientras que las actitudes positivas de los docentes pueden alentar el establecimiento de políticas escolares y la asignación de recursos para aumentar la calidad de la educación de los estudiantes con NEEAD en las aulas regulares (Angenscheidt B \& Navarrete A, 2017).

Muchas dificultades de comunicación experimentadas por los estudiantes con NEEAD pueden resolverse, parcial o totalmente, prestando atención a factores específicos de cada patología que requieren especial atención por parte de los docentes para que estén conscientes de ellas y el trabajo les resulte menos complejo, también sugirieron que los docentes de estudiantes bajo estas condiciones en las aulas regulares deben tener una mejor comprensión de cómo se produce el aprendizaje y de cómo reconocer el progreso (Escareño C, 2017). De lo contrario, los objetivos para la educación de los estudiantes con NEEAD deberían ser los mismos que para los niños que no presentan discapacidad. 
Es importante que los docentes de estudiantes con alguna condición de discapacidad comprendan que la calidad de los entornos en los que los niños con discapacidades aprenden y las expectativas que se generan en ellos, mientras aprenden, son importantes para determinar los logros de aprendizaje individuales (Muñoz C \& Losada P, 2018). Aunque la mayoría de los docentes de clase apoyan los objetivos generales de inclusión, a menudo perciben que la realización de estos objetivos es problemática. La inclusión comienza con su aceptación por parte de los docentes, cuya experiencia y expectativas deberían ser que los niños con NEEAD no necesitan solo escuelas especiales, y la creencia de que el aprendizaje en las escuelas regulares puede ser apropiado para las necesidades de estos niños (González, et al, 2017).

La educación de los estudiantes con NEEAD en clases regulares requiere una planificación y apoyo calificados y reflexivos para una inclusión efectiva para desarrollar las habilidades de comunicación y lenguaje de los estudiantes, de lo contrario, puede contribuir a un rendimiento reducido, falta de atención individual o dificultades personales (Velasquez, et al, 2018). Por lo tanto, los docentes de estudiantes con NEEAD deben tener las habilidades y el conocimiento apropiados de los procesos curriculares inclusivos y simpatizar con los principios de inclusión, además de conocer los tres tipos de adaptaciones curriculares para este tipo de casos: (1) Adaptaciones de Área, (2) Adaptaciones que se realizan en el centro educativo y (3) Adaptaciones que se realizan en el estudiante DIAC. Es probable que las actitudes negativas entre los docentes generen sentimientos negativos similares entre otros docentes y también en la escuela (Angenscheidt B \& Navarrete A, 2017).

Según la normatividad para la inclusión uno de los roles principales de los docentes en clase es crear un ambiente de tolerancia y aceptación para los niños con NEEAD. Sin embargo, como se señaló anteriormente, pueden surgir dificultades si los docentes no tienen un sentido de competencia en la enseñanza y un conocimiento de las estrategias que pueden promover la inclusión de estos estudiantes en la clase y dentro de las actividades escolares a través de experiencias positivas (Arap, 2017).

Es a través de tales experiencias, que los niños pueden llegar a comprender sus propias fortalezas y debilidades y desarrollar capacidades que les permitan superar sus deficiencias para mejorar en su desarrollo académico, personal y social (Mellado H, et al, 2017). Por otro lado, se sugiere que los docentes de clase deben beneficiarse del contacto regular con colegas que realizan un trabajo similar con el que pueden compartir experiencias y también para actualizar las habilidades profesionales en las áreas específicas de las patologías que adolecen sus estudiantes, así como en habilidades para promover la adquisición del lenguaje, la comunicación y las capacidades motoras finas y gruesas (Muntaner G, et al, 2018). Los docentes de apoyo a menudo requieren capacitación profesional en áreas cruciales como el desarrollo de habilidades de trabajo autosuficiente y estrategias de enseñanza colaborativa para estudiantes con dificultades psicomotrices y físicas de aprendizaje (Rodríguez S, 2018). 
Los criterios expuestos parecen cruciales para que las políticas educativas de inclusión hagan operativos los procesos de inclusión haciéndola eficaz y efectiva. Por lo tanto, en este estudio, se buscó determinar si las actitudes de los docentes hacia la inclusión de estudiantes con NEEAD es positiva y si mejora su nivel a través de procesos de sensibilización, considerando la autopercepción de los docentes en el desarrollo de competencias a partir de sus experiencias personales y la influencia de la normatividad para la inclusión en la educación de niños con NEEAD.

\section{Metodología.}

El estudio sobre la sensibilización docente en procesos eficientes de inclusión educativa es una investigación no experimental, a través del cual se busca el fortalecimiento de las capacitaciones de los docentes para implementar eficientemente procesos que posibiliten una vinculación real de estudiantes con Necesidades Educativas Especiales (NEEAD) en el contexto de la educación formal, para el efecto se sustentó teóricamente los procesos de sensibilización educativa partiendo de un marco referencial sobre el problema tratado, el análisis de un estado del arte y una revisión bibliográfica, obtenidos de la documentación existente en repositorios digitales y revistas científicas indexadas en idioma español y en inglés, se utilizó como elementos de búsqueda las variables en estudio: procesos de sensibilización docente e inclusión educativa. Consultada la bibliografía, se encontraron 80 temas relacionados, de los cuales se escogieron 25 artículos de investigación que se acercaron lo más posible al análisis de las variables en estudio de este trabajo.

Se orientó a la identificación de las barreras docentes que dificultan los procesos de inclusión educativa a estudiantes con Necesidades Educativas Especiales, para la consecución de esta información se aplicó una entrevista a diez docentes de la Unidad Educativa Iberoamérica en la ciudad de Ambato. Para el efecto se estableció un calendario de entrevistas, que se llevaron a cabo de forma personal durante un periodo promedio de 30 minutos por entrevistado, el medio utilizado fue el de video conferencia, se utilizaron diversos medios, dependiendo de las facilidades y la disponibilidad de espacio y tiempo de los docentes.

Los datos obtenidos se sistematizaron utilizando el programa Excel y se propusieron en tablas y gráficos. Por las características de la investigación se utilizó un análisis estadístico descriptivo que permitieron reconocer los obstáculos que los docentes tienen frente a la inclusión educativa de estudiantes con Necesidades Educativas Especiales. Lo resultados obtenidos permitieron la sistematización de la problemática y la generalización de la información para sugerir medidas alternativas de soluciones, los resultados alcanzados se socializaron con el grupo de docentes que participaron en la investigación y se remitió un informe a las autoridades del plantel para que consideraran las sugerencias realizadas. 


\section{Resultados}

Para dar cumplimiento a sus procesos, las instituciones educativas ecuatorianas se sujetan a normas jurídicas y a los requerimientos de los organismos estatales vinculados a la educación, esto se evidencia en la legislación que regula la inclusión para estudiantes con NEEAD. Es así que en la Ley Orgánica de Educación Intercultural, el Articulo 6, Literal "o" expresa que entre las obligaciones del Estado se establece "Elaborar y ejecutar las adaptaciones curriculares necesarias para garantizar la inclusión y permanencia dentro del sistema educativo, de las personas con discapacidades, adolescentes y jóvenes embarazadas", en el mismo artículo en el literal " $\mathrm{r}$ " se establece como obligación "Asegurar que todas las entidades educativas desarrollen una educación en participación ciudadana, exigibilidad de derechos, inclusión y equidad, igualdad de género, sexualidad y ambiente, con una visión transversal y enfoque de derechos".

El artículo 11 de la misma Ley en el literal "J" dictamina que entre las obligaciones de los docentes esta "Elaborar y ejecutar, en coordinación con la instancia competente de la Autoridad Educativa Nacional, la malla curricular específica, adaptada a las condiciones y capacidades de las y los estudiantes con discapacidad a fin de garantizar su inclusión y permanencia en el aula".

Los aspectos más particulares relacionados a la inclusión de estudiantes con NEEAD es regulada por la Normativa de Estudiantes con Necesidades Educativas Especiales, en la cual se establecen las funciones de los establecimientos de educación escolarizada ordinaria. En el Art 14 se establecen las funciones de los establecimientos de educación escolarizada ordinaria, en donde se resalta: la planificación, permanencia , promoción y egreso de las estudiantes con NEEAD; Las adaptaciones curriculares pertinentes para atender a los estudiantes con NEEAD, así como la derivación de los estudiantes con problemas de aprendizaje a las Unidades Distritales de Apoyo a la Inclusión (UDAI) para la evaluación, diagnóstico e intervención y finalmente, las instituciones educativas se encargan del desarrollo de programas para la orientación de padres o representantes de los estudiantes con NEEAD.

En el Art. 15, la normatividad de inclusión, establece las responsabilidades que debe asumir el personal de la Institución educativa con respecto a la inclusión, haciéndose referencia a los siguientes aspectos: Velar por la cultura inclusiva, cumpliendo y haciendo cumplir, las disposiciones emitidas para el efecto, los docentes se harán responsables de desarrollar adaptaciones curriculares en el aula, desempeñarse de forma responsable en base a la diversidad de los estudiantes y de acuerdo a sus necesidades, actuar de acuerdo a las necesidades del grupo promoviendo igualdad de oportunidades, dar seguridad y confianza a los estudiantes para un buen desempeño en el aprendizaje, identificar y considerar los intereses de los estudiantes, crear ambientes favorables para la experimentación y la acción, hacer un análisis de las dificultades y destrezas de los estudiantes en forma individual para promover aprendizajes y la participación en las actividades de aula y escolares, detectar los problemas y además detectar los problemas y derivarlos a las UDAI, para la respectiva evaluación y atención de la Unidad. 
Con el propósito de que los docentes desarrollen las habilidades y capacidades descritas en la normatividad, El Ministerio de Educación del Ecuador desarrollo un instructivo denominado; Modelo Nacional de Gestión y Atención para Estudiantes con Necesidades Educativas Especiales Asociadas a la Discapacidad de las Instituciones de Educación Especializadas (Aimacaña, et al, 2019). Este documento está dividido en cuatro componentes, el primero hace una remembranza histórica de los modelos psicopedagógicos utilizados para la integración en la educación, se habla de la estructura del modelo inclusivo de derechos la situación actual de la educación especial en el Ecuador y los trabajos que se han realizado para la atención educativa a la población con discapacidad.

El componente 2 explica los aspectos legales y teóricos, principios y objetivos de la educación especializada, en esta parte del documento resulta importante resaltar que se abarca a todo el conjunto de normatividad jurídica vinculada a la educación inclusiva, pero es tratada de forma sucinta y limitadamente explicativa. La parte de teorización de la inclusión educativa se reduce a elementos y aspectos superficiales y tratados como tips informativos, el aspecto más relevante en este segundo componente es la determinación de la población objetiva que de forma directa refiere a los estudiantes de todos los niveles con NEEAD, y de forma indirecta a todos los miembros de la comunidad educativa, establece que el modelo toma como base los enfoques de derechos, inclusivo, pedagógico, interculturalidad, intergeneracional y de género.

Los principios sobre los cuales se sostiene el modelo se basan en una "educación especializada que debe promover la Autonomía, la Autodeterminación y la Independencia de sus beneficiarios y mejorara la calidad de vida, cumpliendo con lo estipulado por el marco legal vigente" (Aimacaña, et al, p. 41 2019). Plantea como principal objetivo garantizar los derechos de las personas con discapacidad que se encuentran en las instituciones especializadas, respondiendo al contexto social y cultural del grupo de atención prioritario, para lograrlo se propone establecer los lineamientos de gestión organizacional de IEE, establecer una ruta de atención para el acceso, la permanencia y culminación del proceso educativo y propone el establecimiento de los lineamientos técnicos necesarios para la implementación de un currículo nacional con perspectivas de desarrollo ecológico funcional (Ibid, p. 42 2019).

El componente 3 del Instructivo del modelo establece la Estructura Orgánica Funcional, con carácter de dependencia y teóricamente desconcentrado. Respeta la organización estructural del modelo educativo del cual depende, partiendo desde un nivel Central al que se someten los niveles: Zonal, Distrital y Circuital, a cada uno de ellos se le asignan atribuciones para planificar, coordinar y ejercer control en función de las competencias legales asignadas a cada uno de ellos.

El componente 4 del instructivo se centra en proponer especificaciones pedagógicas curriculares para instituciones educativas especializadas (IEE), explicando la aplicación del currículo nacional obligatorio en IEE, los niveles de los niveles de concreción curricular macro, meso y microcurricular, explica la forma de vincular la planificación en 
estos niveles a las NEEAD y hace referencia a aspectos de evaluación y titulación, todo ello entendiéndose como lo que hay que hacer, pero no la forma de hacerlo, seguramente porque el documento fue pensado y diseñado para ser utilizado como un instrumento guía en la formación presencial de los docentes, por parte de la UDAI.

\section{Criterios de los Docentes.}

Con base al contexto jurídico y técnico para la Inclusión Educativa, se realiza un análisis de la sensibilización docente en los procesos de inclusión educativa, considerando los roles institucionales y la participación de los docentes en un proceso complejo para ello se consideraron los criterios de 10 docentes que han sido parte de procesos de inclusión educativa y cuyas experiencias son significativas para comprender el fenómeno desde la perspectiva de la cotidianidad y de las reales necesidades.

\section{Sobre el Rol de la Institución}

Sobre la consideración de que las Instituciones Educativas brindan información y orientaciones para favorecer la inclusión progresiva de los estudiantes con NEEAD, reportan los docentes que es una obligación institucional hacerlo, por lo tanto, de una u otra manera conocen los pormenores de los procesos de inclusión educativa, arguyen que son temáticas tratadas desde hace mucho tiempo atrás, pero que, paulatinamente se han ido vinculando a la actividad cotidiana, haciéndose más intensa la discusión al interior de la institución cuando se presentan este tipo de casos, que cada vez son más frecuentes y con mayor cantidad de estudiantes con NEEAD por aula y con diferentes discapacidades.

Asentir que las Instituciones Educativas Informan a los docentes para favorecer la inclusión progresiva de los estudiantes con NNE, implica las formas en que se da la información, al respecto, siete de los diez docentes aseguraron que están recibiendo capacitación, pero no establecen la manera, tiempos o circunstancias en las que se hace, tres docentes son más puntuales, aseguran que la capacitación que reciben, se realiza generalmente cuando se está estructurando la planificación curricular, con el propósito de integrar los procesos pertinentes y las adaptaciones curriculares necesarias al determinarse la presencia de estudiantes con NEEAD, No se hace referencia a la participación de los UDAI, para solventar estas inquietudes y dar los lineamientos respectivos. Lo que se asegura es que se han contratado docentes especiales con conocimientos en el manejo de estrategias para la inclusión que se encargan de facilitar los procesos.

Las acciones y orientación de las instituciones educativas para lograr la inclusión con la diversidad de estudiantes, de acuerdo a la opinión de los docentes debe tener un enfoque en educación en valores y la búsqueda del trato igualitario sin discriminación de ningún tipo. En este entorno de democratización multidimensional, el mayor peso de la responsabilidad recae en la autoridad que buscara según el criterio de los docentes involucrase eficientemente en hacer cumplir las políticas inclusivas, uno de los docentes encuestados propone literalmente lo siguiente: 
E3: La autoridad educativa del establecimiento se involucra activamente con toda la institución para promover, desarrollar y utilizar estrategias que respondan a las necesidades educativas especiales de sus estudiantes, aspecto trascendental para implementar las políticas inclusivas.

La adopción de los mecanismos de inclusión educativa, sean estos acciones, estrategias u orientaciones se deberán valer de herramientas operativas (Sánchez, et al, 2017), situación de la cual, los docentes tienen plena conciencia, sugiriendo que las capacitaciones sobre inclusión educativa sean programáticas y que estén de acuerdo a las reales necesidades de los estudiantes y las capacidades y potencialidades de los docentes. Además, sugieren los encuestados que debe integrarse un equipo multidisciplinario que este dirigido por un compañero guía que se especialice en los aspectos relacionados a la inclusión. Consideran los docentes la importancia de promover actividades, en las que todos los miembros de la comunidad educativa puedan participar activamente generando de esta manera espacios de encuentro y facilitar los procesos.

Otra de las acciones fundamentales que debe promover la institución para la inclusión son las adaptaciones curriculares pertinentes que permitan que el proceso sea factible, sin embargo, de acuerdo a los docentes entrevistados, este aspecto es una de las dificultades más complejas de solucionar, debido a que las estrategias deben ser sistemáticamente estructuradas y metodológicamente aplicables, por otro lado implica el acondicionamiento de espacios y la adquisición de materiales adicionales con características funcionales adaptadas a los estudiantes estándar y aquellos con NEEAD.

El estado ecuatoriano a través del Ministerio de Educación creó las Unidades Distritales de Apoyo a la Inclusión (UDAI), este organismo, estaría encargado de los procesos de fortalecimiento institucional y docente, para que los centros educativos tengan la operatividad necesaria para incluir en sus aulas de forma eficiente a estudiantes con NEEAD, sin embargo, las limitaciones de presupuesto, la excesiva burocratización en la educación y otros factores coyunturales no han permitido que esta propuesta se desarrolle con la intensidad que debería, de hecho, todos los encuestados aseguran que desde que tienen conocimiento de la existencia de las UDAI, nunca han sido visitados.

Han sido las instituciones educativas, por su propia iniciativa que han desarrollado planes de acompañamiento, capacitación, supervisión, talleres y otros mecanismos de asistencia que han permitido la inclusión educativa. Por el alto nivel de improvisación estas iniciativas no han tenido los efectos esperados, sin embargo, aseguran los docentes que estas acciones de alguna manera han fortalecido sus capacidades.

E5. Todos estos procesos me parecen adecuados ya que hoy en día se impulsa de gran manera la inclusión y los docentes al disponer de la información y conocimientos adecuados se sentirán más capaces de enfrentar los diferentes desafíos que se puedan presentar en este ámbito.

La formación docente para la inclusión, tiene aún un largo camino que recorrer en el sistema educativo ecuatoriano, las buenas intenciones, no cumplen con las expectativas, 
que una organización técnica, bien planificada y adecuadamente asesorada puede alcanzar. Se espera que con el aumento de estudiantes con NEEAD matriculados en la educación formal se fortalezcan los procesos de mejoramiento de las capacidades docentes por parte de las instituciones y los organismos estatales competentes.

Aceptar la inclusión educativa, requiere cambios curriculares, que como se ha explicado con anterioridad, son complejos de lograr en un sistema educativo en el que la malla curricular se ajusta obligatoriamente a los condicionamientos de los organismos burocráticos reguladores. Surgen de esta manera una serie de contradicciones exegéticas, por un lado, se dispone fortalecer los procesos, pero las exigencias de planificación, aplicación, organización y promoción de los elementos curriculares deben ser cumplidos atendiendo a un modelo estandarizado. De acuerdo al criterio de los entrevistados, el acompañamiento y supervisión limita los ajustes estratégicos, debido a que ya están preestablecidos, las adecuaciones son hechas sobre la marcha, basadas en las iniciativas de los docentes que en la mayoría de casos no son reconocidas, y muchas veces sancionadas, esto provoca un alto nivel de apatía. Aseguran los docentes que el acompañamiento es importante, pero es necesario que exista mayor flexibilidad.

E7 Modificar los procesos de aula, para que los estudiantes con necesidades especiales se adapten resulta complicado, hay que cambiar la metodología para que ellos entiendan, pero también para que los estudiantes que no presentan NEEAD no se atrasen, sería bueno que desde la administración educativa se comprendiera este problema y se dieran las pautas para hacer bien las cosas, y no se nos limite.

Los docentes reciben acompañamiento y supervisión permanente, pero no se separa para ello los procesos formales de los que deben adaptarse a las condiciones propias de los estudiantes con NEEAD. Los órganos reguladores y de apoyo como las UDAI deben actuar desde sus competencias dando pautas y lineamientos, proporcionando herramientas y recursos, capacitando a los profesores en función de las necesidades específicas de sus aulas, ya que las generalizaciones provocan confusión, es fundamental que se logren acuerdos y se definan con claridad las estrategias. Por su parte la administración educativa en las instituciones, debe ser puntual y técnicamente realizada, evaluando adecuadamente las acciones que realizan los docentes en su esfuerzo de sensibilizarse, en consonancia con los mandatos vinculados a la implementación de estrategias para la inclusión educativa, que debe trabajarse desde los primeros años de aprendizaje.

Como parte del proceso de acompañamiento los docentes han recibido capacitaciones sobre los lineamientos más generales de la inclusión educativa, esto ha hecho posible que tengan una idea general sobre el tema, por otro lado, se les ha comentado y han discutido sobre los avances que las nuevas tendencias de la educación aplicada a la inclusión ha desarrollado en los últimos años. Sin embargo, el aprendizaje se ha ido desarrollando y consolidándose sobre la marcha, la cada vez mayor presencia de estudiantes con NEEAD y las exigencias de la organización educativa para darles a este grupo de estudiantes un 
tratamiento especial, pero vinculante con los procesos de aula, ha obligado a los docentes que enfrentan este tipo de situaciones a preparase de forma individual, gestionando sus propios conocimientos y de forma general a solicitar asesoramiento a los departamentos de bienestar estudiantil, que de acuerdo a los criterios de los docentes entrevistados, tampoco están adecuadamente preparados para solventar este tipo de situaciones.

E3 El aprendizaje, es permanente, cada caso es diferente, no solo por el hecho de enfrentar una discapacidad $\mathrm{u}$ otra, sino por las diferencias individuales $\mathrm{y}$ psicosociales de los estudiantes, cada estudiante es un mundo y reacciona de diferente manera a los procesos de aprendizaje.

La gestión para la participación de intercambios de experiencias que se realiza para mejorar las capacidades docentes para la inclusión educativa no difiere mucho de la que se realiza para los procesos formales de aprendizaje. Las actividades de encuentro interinstitucional abarcan una gran diversidad de temáticas, de entre las cuales, se ha tocado también el tema de la inclusión educativa. Se discuten, como ya ha quedado dicho con anterioridad, los temas más generales y no se realizan intercambios de ideas y peor aún sistematización de experiencias. La actitud de los docentes es más bien de un intercambio de experiencias desde la perspectiva del compañerismo, la amistad y el deseo de ayudarse mutuamente para solventar las dificultades, así lo expresan dos de los entrevistados:

E5 Generalmente, cuando le toca a uno un estudiante con NEEAD, se enfrenta a una situación desconocida, en este caso lo que se hace es preguntar a los compañeros, quién ha pasado por una experiencia de ese tipo y averiguar de qué forma, el compañero, pudo salir adelante.

E9 ...conversamos entre compañeros de la misma institución o de otras instituciones y preguntamos quienes han tenido casos de estudiantes con NEEAD, entonces compartimos las experiencias y vamos solventando los problemas sobre la marcha... Siempre es importante conversar con otros compañeros, acoger las ideas e intercambiar experiencias.

\section{Sobre el Currículo}

Es tarea del Departamento de Consejería Estudiantil (DECE), junto a los docentes que manejan estudiantes con NEEAD, vincular el currículo programado a los casos que se presentan en la institución de forma general y en cada aula de forma particular. Este proceso se hace de diversas maneras, generalmente adaptándose a las situaciones que se presentan en los entornos, se consideran las experiencias curriculares que previamente se hayan tenido, y se hacen las adaptaciones curriculares pertinentes en función de los requerimientos específicos de los estudiantes y las capacidades operativas de las instituciones, dentro de las cuales se deben considerar muchos factores que dependen de las características de los casos. 
La inclusión educativa, es un problema complejo al momento de vincular las estrategias para el manejo de estudiantes con NEEAD con la planificación curricular (Aguinaga, et al, 2018). Sobre la base de una planificación estructurada para formar estudiantes con capacidades estandarizadas y tomando en cuenta la rigidez de la enseñanza por sus contenidos obligatorios y metodologías pre diseñadas, además del gran peso burocrático que las disposiciones gubernamentales han puesto sobre los docentes. Adaptar la planificación curricular se vuelve una tarea compleja, los docentes entrevistados aseguran que a nivel institucional se designa a los profesores para que sean ellos quienes den solución a la planificación que requiere el estudiante, se pone a su disposición el DECE como apoyo y se confía a su criterio las acciones que se deben realizar para enfrentar los problemas que conlleva la inclusión educativa

En las conversaciones realizadas con los docentes proponen las siguientes soluciones, en primer lugar se debe hacer un análisis exhaustivo del caso y evaluar si la institución tiene las condiciones necesarias como para enfrentar y solucionar las dificultades de los estudiantes con NEEAD; en segundo lugar es necesario que a los docentes se les capacite de forma específica, esto es que deben existir procesos de formación docente vinculados directamente con las necesidades precisas del estudiante con NEEAD; como tercer punto, es necesario que exista un nexo permanente y un compromiso en firme con los representantes de los estudiantes, con el propósito de que las acciones emprendidas sean consecuentes con sus responsabilidades; por otro lado deben quedar claros los lineamientos de la intervención educativa a la que se enfrenta el docente, así como el alcance que es posible lograr técnica y racionalmente con el estudiante de acuerdo a sus potencialidades y nivel de desarrollo; finalmente las instituciones deberían respaldar plenamente el trabajo de los docentes dándoles el apoyo necesario sin exigir de ellos cosas que están fuera de su alcance como profesionales y como seres humanos.

\section{Sobre el apoyo a los estudiantes con Necesidades Educativas Especiales}

El apoyo a los estudiantes con NEEAD, es un proceso divergente que carece de una estructura sincrónica adaptada a la planificación curricular tradicional, la administración pedagógica institucional trata de encontrar los mecanismos más idóneos, pero en última instancia son los docentes quienes asumen el reto de formarlos. Para lograr este propósito se han adoptado medidas que se ajustan a los recursos disponibles en la institución y a los espacios de enseñanza asignados para dar solución a los problemas de rendimiento de toda la población de estudiantes,

E5. ... se da en un horario fuera de la jornada de clases en donde se le da al estudiante un apoyo pedagógico y un refuerzo académico personalizado con el fin de impartir el conocimiento de una manera más personalizada y eficaz.

El refuerzo del aprendizaje fuera de las horas normales de clase, es la alternativa que los docentes utilizan para tratar de solventar las nesecidades educativas de los estudiantes con discapacidad, aceptan los docentes que deben trabajar horas adicionales para reforzar los contenidos, pero como se ha dicho estos procesos se van realizando alternativamente 
y sobre la marcha, no existe una planificación especificamente estructurada para este tipo de acciones.

Por otro lado, es necesario hacer notar que la inclusión implica la participación de todos los actores del proceso del aula, la mayor parte de las acciones deben estar orientadas al trabajo colaborativo en el cual los estudiantes con NEEAD, se sientan parte del grupo, al considerar la alternativa de los espacios de refuerzo dentro y fuera del aula, al respecto uno de los docentes entrevistados propone:

E6. La inclusión debe promover programas de nivelación, refuerzos dentro y fuera del horario habitual.

El alcance del apoyo de los docentes a los estudiantes con NEEAD, concretamente se da en función de las potencialidades que cada uno tiene para lograr la inclusión en sus aulas y a través de los refuerzos ocacionales que se dan como apoyo pedagógico, en estas acciones los docentes hacen un gran esfuerzo asumiendo una mayor cantidad de responsabilidades que los docentes que no tienen estudiantes con NEEAD en sus aulas.

Este hecho hace necesaria una revisión exaustiva de los procedimientos que se emplean en los centros educativos para fomentar los procesos de inclusión, para que el apoyo que realizan los profesores a los estudiantes con discapacidad sea más eficiente. Muchos y diversos son los factores que influyen en los programas de apoyo en las tutorias fuera del horario escolar, sin embargo, se debe resaltar la necesidad de establecer institucionalmente politicas consistentes y a mediano y largo plazo que posibiliten al docente formarse adecuadamente y adaptarse de forma progresiva a los programas.

Otro aspecto a considerar, son los recursos didácticos a los que los docentes tienen acceso, generalmente las instituciones educativas trabajan en función de las necesidades de la mayoría de los estudiantes, y se adquieren o solicitan a los organismos competentes los recursos que pueden ser de utilidad para la mayoría. De esta forma se discriminan las necesidades específicas de los estudiantes con NEEAD, de ahí que las instituciones deben asignar presupuestos especiales para la consecución de materiales adecuados y adaptados a las diferentes necesidades que la inclusión implica.

Los programas de refuerzo para los estudiantes con NEEAD, deben ser adecuadamente planificados, debido a que se van más allá de las competencias habituales que manejan los docentes, por lo que se deben trabajar con mucho más cuidado y bajo la supervisión permanente de personal que maneje de forma adecuada los procedimientos.

Las acciones de recuperación, más que en el refuerzo de contenidos, deben estar orientadas al desarrollo de habilidades y destrezas que le permitan al estudiante con NEEAD, desempeñarse lo más fluidamente posible en el contexto de las interrelaciones con sus pares, con los docentes y de forma general con todos los actores del proceso educativo, no es importante insistir en el aprendizaje de contenidos, sino más bien en potenciar las habilidades de aprendizaje individual y colectivo del estudiante con NEEAD hasta lograr su autonomía e independencia. 
El rigor de los aprendizajes en programas de apoyo y recuperación implican un mayor desgaste para el docente y los estudiantes, por lo que las actividades que se desarrollen deberán ser más informales y relajadas, además de que debe existir la participación efectiva de los representantes de los estudiantes que deben apoyar física y emocionalmente a las iniciativas propuestas en este tipo de intervención y ser un soporte permanente y no un obstáculo, aceptando la discapacidad de su representado y apoyando en el proceso de enseñanza - aprendizaje.

\section{El Rol de los Padres de Familia.}

La presencia activa de los padres de familia en los procesos de inclusión es fundamental para alcanzar los mejores resultados, su participación inicia proporcionando a la institución educativa la información precisa que permite identificar con claridad las condiciones en las que ingresa el estudiantes con NEEAD, esto es, el tipo de problema que tiene, cual ha sido el diagnostico que le han proporcionado los médicos tratantes, cuáles son los tratamientos médicos y psicoterapéuticos que debe seguir, que experiencias de aprendizaje ha tenido, cuales han sido los resultados, entre otros factores. Uno de los docentes declara en la entrevista realizada que:

E1. Los padres de familiares se encargan de transmitirnos los informes de los exámenes y pruebas emitidas por el departamento de consejería, mediante el cual los docentes se encargan de realizar las adaptaciones curriculares que cubran las necesidades de aprendizaje de cada uno de los estudiantes, creando así una educación individualizada y personalizada.

Generalmente los padres de familia, al acercarse a la institución educativa, son sujetos de una entrevista que se lleva a cabo en el DECE, se llena un formulario en el que se establece si el estudiante tiene algún problema vinculado a NEEAD, cuando el departamento establece la situación asigna a un Psicólogo educativo para que realice una entrevista especial con los padres del estudiante y en esta reunión los padres deberán entregar toda la documentación correspondiente al caso del estudiante y demostrar a través de pruebas y exámenes actualizados la condición médica y psicológica.

Si el estudiante proviene de otra institución educativa, la institución de origen está obligada a emitir un informe del desarrollo de aprendizajes, comportamiento, nivel de adaptabilidad y de inclusión que el estudiante logró en esta institución de origen, en casos excepcionales, Los DECE de las dos instituciones, bajo el auspicio de un arbitraje generalmente garantizado por el UDAI, conversan sobre la situación del estudiante.

Bajo estos parámetros los padres de familia deben colaborar eficientemente con las instituciones educativas que acogen a su representado con el propósito de llegar a acuerdos y solventar los mecanismos necesarios para que el estudiante con NNE especiales pueda tener la mejor atención posible por parte de los educadores y estos a su vez tengan los elementos de juicio para ayudar eficientemente a los estudiantes. 


\section{Sobre los recursos institucionales}

Uno de los factores que reduce los procesos de inclusión en las instituciones educativas son los recursos físicos y la infraestructura. De esos aspectos se habla muy poco, pero son los principales argumentos que las instituciones educativas utilizan para evadir su responsabilidad de la educación inclusiva. Los establecimientos de las instituciones educativas, fueron construidos con mucha antelación a la idea de considerar a la inclusión de estudiantes con NEEAD, como parte de sus programas, por lo que la infraestructura, generalmente está diseñada para cumplir con los requerimientos estandarizados para la educación formal, por lo que es necesario que se establezcan estrategias para solucionar el ingreso de estudiantes con diversas discapacidades físicas y sensoriales formal.

Por otro lado, están los recursos pedagógicos y didácticos, que en el sistema educativo ecuatoriano apenas si cubren las necesidades para el aprendizaje de los estudiantes formales, esto es laboratorios con limitado equipamiento, materiales didácticos descontinuados, incompletos, desgastados o averiados, espacios de recreación afectados por el continuo uso, áreas de deporte en malas condiciones, entre otras situaciones.

Bajo esta realidad, la adaptación de los espacios y recursos para la inclusión educativa resulta una inversión alta en relación al beneficio global que los cambios podrían generar en los procesos de aprendizaje, de ahí que la administración educativa de cada plantel piense muy detenidamente para realizar cambios en las infraestructuras, sin embargo, al aceptar estudiantes con NEEAD, es una realidad que tiene que ser afrontada.

Desde esta perspectiva es importante que en los presupuestos anuales que se les entrega a las instituciones educativas públicas se asignen rubros para el mejoramiento de los espacios requeridos por los estudiantes con discapacidad de igual manera, las instituciones fiscomisionales y privadas deben realizar las gestiones necesarias para la consecución de recursos, que posibiliten la adecuación de los espacios de aprendizaje. En la entrevista realizada el criterio de los docentes demuestra una buena predisposición para el trabajo, aseguran que las instituciones educativas pueden recibir a estudiantes con NEEAD, siempre y cuando se hagan las adecuaciones pertinentes:

E5. En este aspecto la institución cuenta con las medidas necesarias para la cantidad de estudiantes que tienen NEEAD, sin embargo, al ser un espacio limitado necesita la implementación de rampas si en el futuro se presenta un niño con discapacidad motriz.

Las necesidades de los centros educativos con respecto a los procesos de inclusión, están directamente relacionadas con la cantidad y el tipo de estudiantes con NEEAD que reciben en sus aulas. Las características de los estudiantes con NEEAD son diversas, y por lo tanto requieren de espacios adaptados para las diferentes discapacidades, lo que hace compleja la tarea de los planificadores administrativos para tomar la decisión de por dónde empezar por los cambios, al respecto los docentes sugieren que se debería realizar un censo educativo en los espacios territoriales, establecer la cantidad de estudiantes con NEEAD y cuáles son esas necesidades, evaluar las instituciones educativas y asignarles 
uno o dos tipos de problemas, con el propósito de hacer más factible la operatividad de las instituciones, uno de los entrevistados habla con mucha claridad al respecto:

E9 Se debe realizar un sondeo de la cantidad de potenciales estudiantes con NEEAD en el sector, como existen diferentes instituciones educativas, a cada una se le debe especializar en un tipo de condición, por ejemplo, una institución podría hacer inclusión con estudiantes ciegos, otra institución con estudiantes que utilizan sillas de ruedas, otra institución con estudiantes que presenten discapacidad intelectual, etc.

De esta manera, aseguran los entrevistados, las instituciones optimizarían sus recursos, la preparación de los docentes sería más eficiente y los costos de implementación menores. Esta resulta una interesante sugerencia para la organización de la inclusión en los procesos educativos, pudiendo ser una alternativa al problema de déficit de facilidades en las instituciones, además de disminuir la presión en la organización y planificación, tanto de los centros educativos como de los docentes. La especificidad de atención en los centros educativos para los estudiantes con NEEAD, se presenta como una alternativa importante que debería ser analizada y discutida como una posibilidad en el complejo problema de la inclusión educativa que vive el sistema de enseñanza en el Ecuador.

\section{Discusión}

Los cambios que genera la inclusión en los procesos educativos formales conllevan la investigación y el análisis de las propuestas metodológicas y organizativas que vinculadas a la predisposición de los docentes paulatinamente responden a la realidad de las aulas, en este contexto el trabajo precedente hace referencia a aspectos relevantes en el rol de las instituciones educativas y de los docentes en busca de la sensibilización para la inclusión. Presenta interesantes aportes en un área de investigación en la que las investigaciones puntuales son escasas. Del criterio de los docentes se han obtenido aportaciones originales e importantes las cuales son contrastados con datos de otros trabajos, se propone a continuación los resultados derivados de este estudio.

Con respecto a las estrategias institucionales para la inclusión educativa, esta investigación encuentra que un factor fundamental para la toma de decisiones es tener en cuenta la estructura jurídica que regula los procesos sociales y educativos y que proporciona los lineamientos necesarios para determinar los niveles de responsabilidad y las competencias que les son inherentes a cada uno de los actores educativos, al respecto Arellano y Súarez (2019) señalan que:

...la participación de las organizaciones sociales es necesaria para la sensibilización y el apoyo a los centros educativos, sin embargo se presentan dos obstáculos el reconocimiento de las organizaciones como coadyuvantes de los procesos de sensibilización y de capacitación y la falta de empoderamiento de la sociedad civil, lo cual, no es una cuestión de organización, sino del desconocimiento jurídico que determina el papel de los ciudadanos y los organismos competentes en el caso de la inclusión educativa. 
Los docentes entrevistados, que participaron en el estudio reflejan una posición positiva con respecto a la inclusión de estudiantes con NEEAD, y demuestran una predisposición favorable para integrase a los planes y programas institucionales que impliquen el mejoramiento de los entornos de aprendizaje para beneficiar a los estudiantes discapacidad. Estos criterios coinciden con los reportes de otros trabajos de investigación como: (Castro, et al, 2016; Angenscheidt et al, 2017; Acosta y Arráez, 2017; Fernández B, 2018; Lalama F, 2018; Palacio S, 2019; Miranda et al, 2020), quienes reportan, de los docentes con los que realizaron sus trabajos de investigación, una actitud favorable hacia la inclusión y destacan la importancia de los beneficios que este proceso puede generar en la formación de los estudiantes.

Romero y otros autores (2019) aseguran que, para la adopción de un determinado nivel de inclusión educativa, es importante considerar el status operativo de la institución y las capacidades adquiridas de los docentes. También, Sánchez junto a otros investigadores (2017), aseguran que para adoptar la inclusión educativa es necesario contar con herramientas operativas que permitan dar viabilidad a las acciones necesarias para la inclusión. En esta investigación la información obtenida revela que las necesidades requeridas para la inclusión educativa tienen su soporte en una intervención programática, en las reales necesidades de los estudiantes y las competencias que han adquirido los docentes. Esto se suma a las modificaciones curriculares y procedimientos que se deben incorporar, todo ello deberá consolidarse a través de una administración operativamente viable a través de herramientas funcionales.

Pegalajar y Colmenero (2017), consideran que la inclusión educativa funciona como un factor coadyuvante entre las actitudes y la disposición que se dan en la diversidad, antes que en acciones concretas y aisladas, lo que implica la reestructuración de la organización educativa con el propósito de ofrecer una acertada atención educativa a todos los estudiantes, de ahí que el docente es un elemento clave en la construcción de la inclusión educativa, resulta imposible avanzar en los procesos sin la participación efectiva de los profesores, porque son ellos quienes asumen directamente el trato con los estudiantes con NEEAD. Al respecto se ha encontrado que los docentes no reniegan su responsabilidad, sin embargo, están conscientes que la inclusión educativa implica mayor trabajo y que sus esfuerzos deben ser reconocidos y valorados.

Se ha determinado que a pesar de la existencia de variables que inciden en la respuesta de los docentes, asumen la responsabilidad con un elevado compromiso profesional, demostrando que no existe una respuesta negativa a la inclusión y que se manifiestan de forma positiva contribuyendo altitudinalmente con su disposición al trabajo en favor de los estudiantes con NEEAD.

Considerando los resultados de esta investigación se establece que los docentes entrevistados demuestran predisposición para fomentar la inclusión educativa, sin embargo, requieren de mejor formación, recursos didácticos, y entornos pedagógicos adecuados. A pesar de los limitados medios con los que cuentan los docentes utilizan frecuentemente estrategias inclusivas en los procesos de aula, que se aplican de acuerdo 
a la variabilidad de los casos de los que son responsables. Bajo este contexto se puede asegurar que existe una relación directa entre las actitudes positivas de los docentes con la calidad y la cantidad de estrategias que utilizan. Esto quiere decir que mientras exista una mayor sensibilidad para la inclusión educativa por parte de los docentes, estos buscaran un mayor número de mecanismos para hacer eficiente el aprendizaje con los estudiantes con NEEAD. Por lo tanto, queda demostrada la idea a defender de que la eficiencia en la inclusión educativa está determinada por los procesos de sensibilización docente.

\section{Conclusiones.}

- Los resultados obtenidos en esta investigación van más allá de las acciones que realizan las instituciones educativas y los docentes para mejorar la condición de los estudiantes con NEEAD, busca establecer cuáles son las relaciones que se dan en los procesos educativos y de qué manera se ha desarrollado la sensibilización en los actores educativos, por lo tanto se considera una investigación de relevancia para ahondar y determinar las necesidades y requerimientos específicos que implica lograr una actitud positiva frente a la inclusión de calidad.

- La inclusión educativa para los estudiantes con NEEAD es un derecho, los aspectos que son inherentes con su aplicación están estipulados en un cuerpo jurídico que les da operatividad legal. La legalidad implica obligatoriedad, por lo que las instituciones educativas tienen el deber de impulsar estos procesos. Sin embargo, de estar establecidos los lineamientos teóricos de la inclusión educativa, las necesidades y requerimientos objetivos dependen de una gran diversidad de factores que vuelven a la inclusión un proceso complejo.

- Esencialmente, las demandas para la inclusión de las instituciones educativas están relacionadas con el mejoramiento de los espacios físicos de los entornos en los que se desenvuelven los estudiantes incluidos aquellos con NEEAD. Pero sobre todo requieren de una formación programática de los docentes y la potencialización de sus capacidades para mejorar asertivamente los procesos, procurando el éxito de todos los estudiantes en la enseñanza aprendizaje en el aula y lograr un nivel de calidad de aprendizaje homogéneo.

- La generación de estrategias para la implementación de la inclusión educativa depende de varios factores, desde la diferenciación teórica de los términos inclusión educativa con integración educativa que producen confusión, hasta la consolidación de las estrategias diseñadas de forma particular para cada grupo de estudiantes o centradas en los estudiantes de forma particular, así como los mecanismos que utilizan las instituciones educativas para interrelacionar y sistematizar las experiencias significativas.

- La aceptación de la inclusión educativa transita todavía por un sendero tortuoso y complejo, considerando que es un enfoque relativamente nuevo, y que se trató, no hasta hace mucho tiempo, desde la filosofía de la integración educativa que, si bien es un paso para lograr la inclusión y se relaciona con las estrategias y metodologías centradas en el estudiante, no contempla los aspectos funcionales 
que resaltan las capacidades individuales relacionadas con la sociabilización como principio de la educación inclusiva.

- Las dificultades que enfrenta la sensibilización docente para la inclusión, están relacionadas significativamente a los entornos de aprendizaje, y los recursos pedagógicos con los que cuentan las instituciones, que están obligadas a realizar trasformaciones en infraestructura externa y de aula, así como la sustitución de materiales y el acondicionamiento o potenciación de los recursos de enseñanza para adaptarlos a las NEEAD. este problema influye en la capacidad de receptividad de estudiantes y en las facilidades que se le puede dar al docente para realizar mejor su trabajo.

- Investigaciones futuras y el desarrollo de planes y programas basados en resultados permitirán solventar con mayor certeza los problemas de los docentes haciendo posible detectar con eficacia las necesidades y proponer el tipo de apoyo y la formación requerida para los procesos de inclusión, y si estas son eficaces para el cambio de actitudes de los docentes hacia la sensibilización.

\section{Referencias bibliográficas.}

Acosta , A., \& Arráez , T. (2017). Actitud del docente de educación inicial y primaria ante la inclusión escolar de las de las personas con discapacidad motora. Revista de Investigación, 137 - 154.

Aguinaga, S., Velázquez, M., \& Rimari, M. (2018). Modelo contextualizado de inclusión educativa. Revista educación, 42(2), 109-126.

Aimacaña , J., Espinoza , T., Pérez, D., \& Viteri, C. (2019). Modelo nacional de gestión y atención para estudiantes con Necesidades Educativas Especiales Asociadas a la discapacidad de las Instituciones de educación Especializadas. Quito: Ministerio de Educación del Ecuador.

Angenscheidt B, L., \& Navarrete A , I. (2017). Actitudes de los docentes acerca de la educación inclusiva. Ciencias Psicológicas, 11(2), 233-243.

Arap, M. (2017). Actitud docente e inclusión educativa: un estudio de caso sobre la influencia de la actitud en la práctica docente en clases "inclusivas". Montevideo: Asociación civil Incluime.

Arellano, T., \& Súarez, E. (2019). Sociedad civil e inclusión educativa: el reto de las organizaciones de apoyo. Controversias y Concurrencias Latinoamericanas, 10(18), 59-69.

Asamblea Constituyente. (2008). Constitución de la Republica del Ecuador. Montecristi.

Castro , P., Álvarez, M., \& Baz , B. (2016). Inclusión educativa. Actitudes y estrategias del profesorado. Revista Española de Discapacidad (REDIS), 4(2), 25-45. 
Escareño C, J. (2017). Docente inclusivo, aula inclusiva. Revista de Educación Inclusiva, 9(2), 264-275.

Fernández B, J. (2018). Competencias docentes y educación inclusiva. Revista electrónica de investigación educativa, 15(2), 82-99.

García-Cerda, L., Rodríguez-Fernández, O., Betancourt-Galindo, R., Saldívar-Guerrero, R., \& Torres-Torres, M. (2003). Síntesis y propiedades de ferrofluidos de magnetita. Superficies y Vacío., 16(1), 28-31.

González F, R., Palomares R, A., \& Domingo G, B. (2017). Factores desencadenantes de la actitud del profesorado hacia la inclusión educativa. Prácticas innovadoras inclusivas: retos y oportunidades. Políticas socioeducativas inclusivas y Formación del Profesorado, 365-370.

Herrera I, J., Parrilla , Á., Blanco, A., \& Guevara, G. (2018). La formación de docentes para la educación inclusiva. Un reto dUn reto desde la Universidad Nacional de Educación en Ecuador. Revista latinoamericana de educación inclusiva, 12(1), 21-38.

Lalama F, A. (2018). Inclusión educativa:¿ quimera o realidad?. Conrado, 14(62), 134138.

Machado S, M., \& Vintimilla C , M. (2019). La inclusión educativa de los estudiantes con necesidades educativas especiales (NEE): sensorial, intelectual, física y altas capacidades en la educación general básica regular de la zona 1 del Ecuador. C uenca: Universidad del Azuay.

Martínez P, J., \& Moreno P, I. (2018). Inclusión: Un tema de derechos. Revista Boletín Redipe, 7(6), 39-51.

Mellado H, M., Chaucano C, J., Hueche O, M., \& Aravena K, O. (2017). Percepciones sobre la educación inclusiva del profesorado de una escuela con Programa de Integración Escolar. Revista Educación, 41(1), 119-132.

MIES. (2013). Acuerdo Ministerial 295 Registro Oficial 93 Normativa de estudiantes con necesidades educativas especiales. Quito : Lexis .

Miranda V, M., Laz S, M., \& Campuzano P, M. (2020). Inclusión y su importancia en las instituciones educativas desde los mecanismos de integración del alumnado. Revista Arbitrada Interdisciplinaria Koinonía, 5-27.

Montaner G, J., Rosselló R, M., \& Iglesia M, B. (2018). Buenas prácticas en educación inclusiva. Educatio Siglo XXI, 34(1), 31-50.

Muñoz C, J., \& Losada P, L. (2018). Implicaciones de la actitud docente en la calidad de vida del alumnado con alteraciones del desarrollo intelectual. Educación XXI, 21(2), 37-58. 
Olivares G, Á., \& García B, M. (2018). Formación del profesorado de educación inclusiva: reto docente de la educación especial. Opción, 31(3), 584-604. Obtenido de https://www.redalyc.org/pdf/310/31045567030.pdf

Palacio S, J. (2019). Una mirada a los procesos de inclusión escolar. Manizales: CINDES.

Palomino P, M. (2017). Carmen Pegalajar. Importancia de la actividad formativa del docente en centros de Educación Especial. Revista Electrónica Interuniversitaria de Formación del Profesorado, 17(1), 177-192. Obtenido de https://revistas.um.es/reifop/article/view/177

Pegalajar, M., \& Colmenero, M. (2017). Actitudes y formación docente hacia la inclusión en Educación Secundaria Obligatoria. Revista electronica de la Investigación educativa, 19(1), 84-97.

Rodríguez S, C. (2018). Ambientes de aprendizaje colaborativo en comunidades artístico-pedagógicas. MAdrid: Universidad complutense de mADRID.

Romero, C. (2019). La Inclusión educativa comop proceso en contextos socioeducativos. Madrid: UNED.

Sánchez, P., Frutos, E., \& García, M. (2017). El impacto del contexto escolar en la inclusión educativa. Revista de Educación Inclusiva, 10(2), 195-210.

Sevilla S, D., Martín P, M., \& Jenaro R, C. (2018). Cristina. Actitud del docente hacia la educación inclusiva y hacia los estudiantes con necesidades educativas especialesCristina. Actitud del docente hacia la educación inclusiva y hacia los estudiantes con necesidades educativas especiales. Innovación educativa, 18(78), 115-141.

UNESCO. (1994). Declaración de Salamanca sobre Necesidades Educacionales Especiales. Salamanca: UNESCO.

Velasquez U, Y., Quiceno F, E., \& Tamayo A, W. (2018). Construcción de planeaciones pedagógicas para la educación inclusiva. Actualidades investigativas en educación, 16(3), 331-366.

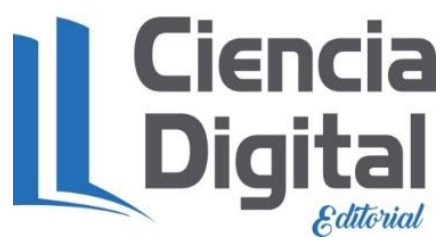




\section{PARA CITAR EL ARTÍCULO INDEXADO.}

Aranda, M. J., \& Jiménez Sánchez, C. del C. (2021). Sensibilización docente en procesos eficientes de inclusión educativa. Explorador Digital, 5(3), 38-60. https://doi.org/10.33262/exploradordigital.v5i3.1751

\section{Liencia}

El artículo que se publica es de exclusiva responsabilidad de los autores y no necesariamente reflejan el pensamiento de la Revista Explorador Digital.

El artículo queda en propiedad de la revista y, por tanto, su publicación parcial y/o total en otro medio tiene que ser autorizado por el director de la Revista Explorador Digital.
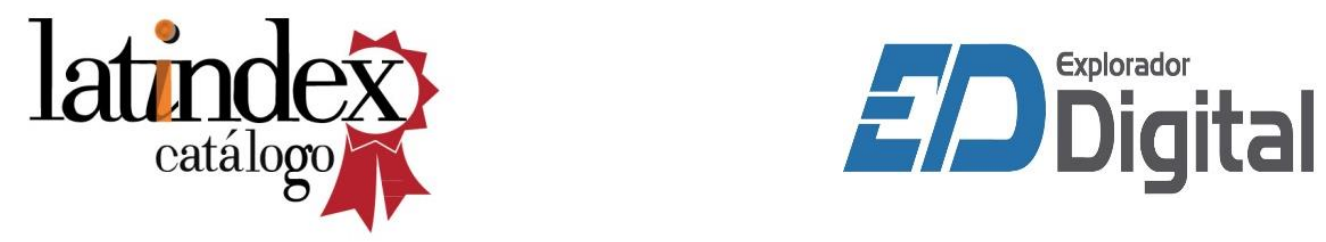